\title{
Probing the MSP prenatal stage: the optical identification of the X-ray burster EXO 1745-248 in Terzan 5
}

\author{
F. R. Ferraro ${ }^{1}$, C. Pallanca ${ }^{1}$, B. Lanzoni ${ }^{1}$, M. Cadelano ${ }^{1,2}$, D. Massari ${ }^{2,3}$, E. Dalessandro ${ }^{1}$, \\ A. Mucciarelli ${ }^{1}$, \\ ${ }^{1}$ Dipartimento di Fisica e Astronomia, Università degli Studi di Bologna, v.le Berti Pichat \\ 6/2, I-40127 Bologna, Italy \\ ${ }^{2}$ INAF-Osservatorio Astronomico di Bologna, via Ranzani 1, 40127, Bologna, Italy \\ ${ }^{3}$ Kapteyn Astronomical Institute, University of Groningen, PO Box 800, 9700 AV \\ Groningen, The Netherlands
}

02 May, 2015

\begin{abstract}
We report on the optical identification of the neutron star burster EXO 1745248 in Terzan 5. The identification was performed by exploiting HST/ACS images acquired in Director's Discretionary Time shortly after (approximately 1 month) the Swift detection of the X-ray burst. The comparison between these images and previous archival data revealed the presence of a star that currently brightened by $\sim 3$ magnitudes, consistent with expectations during an X-ray outburst. The centroid of this object well agrees with the position, in the archival images, of a star located in the Turn-Off/Sub Giant Branch region of Terzan 5. This supports the scenario that the companion should has recently filled its Roche Lobe. Such a system represents the pre-natal stage of a millisecond pulsar, an evolutionary phase during which heavy mass accretion on the compact object occurs, thus producing X-ray outbursts and re-accelerating the neutron star.
\end{abstract}

Subject headings: binaries: close; globular clusters: individual (Terzan 5); stars: neutron; X-rays: bursts; X-rays: individual (EXO 1745-248)

\footnotetext{
*Based on observations (GO 14061, GO 12933, GO 9799) with the NASA/ESA Hubble Space Telescope, obtained at the Space Telescope Science Institute, which is operated by AURA, Inc., under NASA contract NAS 5-26555.
} 


\section{INTRODUCTION}

Low mass X-ray binaries (LMXBs) and radio millisecond pulsars (MSPs) are thought to be, respectively, the starting and the ending stages of a common evolutionary path, where a neutron star accretes matter (and angular momentum) from a companion (e.g., Bhattacharya \& van den Heuvel 1991; Wijnands \& van der Klis 1998). The early phases of this evolutionary path are characterized by active mass accretion accompanied by intense $\mathrm{X}$-ray emission (larger than $\sim 10^{35} \mathrm{erg} \mathrm{s}^{-1}$ ). These systems are observed as LMXBs characterized by a few outburst in the X-ray due to accretion disk instabilities. These objects are usually called "X-ray transients" (White et al. 1984). When the neutron star is reaccelerated, the system will appear as a MSP in the radio band. Newly born MSPs are therefore expected to have a bloated and tidally deformed companion which is still losing mass from its Roche Lobe. In this case, the system is called "redback" (or "black widow" if the companion is less massive than $0.05 M_{\odot}$ ). Following the canonical scenario, at the end of the evolution only the degenerate core of the peeled companion (i.e., a helium or carbon-oxygen white dwarf) is predicted to orbit the MSP (a sub-stellar mass object, which is eventually completely evaporated, in the case of a black widow). Indeed, the optical searches for the companion stars to binary MSPs performed so far in Galactic globular clusters detected objects belonging to all these three classes: five bloated stars, companions to redbacks (see Ferraro et al. 2001; Edmonds et al. 2002; Cocozza et al. 2008; Pallanca et al. 2010, 2013), two very low mass objects companions to black widows (Pallanca et al. 2014, Cadelano et al. 2015, submitted to ApJ), and eight white dwarfs companions to canonical MSPs (Edmonds et al. 2001; Bassa et al. 2003; Ferraro et al. 2003; Sigurdsson et al. 2003, Mario Cadelano et al. in preparation, Liliana Rivera Sandoval et al. in preparation). A new unexpected link has been added to the chain very recently. Papitto et al. (2013) found that the X-ray transient IGR J18245-2452 in the globular cluster M28 (Eckert et al. 2013) corresponds to MSP M28I, and the system is currently swinging between accretion-powered and rotation-powered states. This shows that the transition from a LMXB to a MSP passes through an intermediate phase during which the two states cyclically alternate over a time scale of a few years (Papitto et al. 2013). A low-mass main sequence star, which experienced a strong ( $\sim 2$ magnitudes) luminosity increase, has been identified as the optical counterpart to the system (Pallanca et al. 2013).

On March 13, 2015, Swift/BAT observations detected an X-ray burst in Terzan 5 (Altamirano et al. 2015). The Swift/XRT observations promptly following the Swift/BAT detection localized the transient source at RA $(\mathrm{J} 2000)=267.0207 \mathrm{deg}, \mathrm{DEC}(\mathrm{J} 2000)=-24.779 \mathrm{deg}$, with a $90 \%$ uncertainty of $3.5^{\prime \prime}$ (Bahramian et al. 2015). Moreover, the measured spectrum turned out to be consistent with a relatively hard photon index of $1.0 \pm 0.2$ and a hydrogen column density $N_{\mathrm{H}}=(4 \pm 0.8) \times 10^{22} \mathrm{~cm}^{-2}$. The latter is larger than the typical value 
measured in Terzan 5 (Bahramian et al. 2014) and well in agreement with the hydrogen column density of the previously known transient EXO 1745-248 (Kuulkers et al. 2003). Indeed, the subsequent position refinement by Linares et al. (2015) centered the system around EXO 1745-248 with a 2.2" error circle. These data therefore strongly suggest that the new Swift/BAT outburst coincides with EXO 1745-248, an X-ray neutron star transient that already showed outbursts in 2000 and 2011 (Degenaar \& Wiinands 2012). Such an identification has been also confirmed by radio VLA observations (Tremou et al. 2015), which locate the source position within $0.4^{\prime \prime}$ of the published coordinates of EXO 1745-248 obtained from Chandra data (source CX3 in Heinke et al. 2006). The most recent Swift/XRT observations indicate that the source is probably on the way to transit to the soft state (Yan et al. 2015).

Such an intriguing object is not uncommon in Terzan 5. In fact, this stellar system is known to harbor several X-ray sources (see, e.g., Heinke et al. 2006) and to be the most efficient furnace of MSPs in the Milky Way: it harbors a total of 34 MSPs, corresponding to $\sim 25 \%$ of the entire sample of such objects known to date in Galactic globular clusters (Ransom et al. 2005). Ferraro et al. (2009) recently demonstrated that, at odds with what is commonly thought, Terzan 5 is not a globular cluster, but a system hosting stellar populations characterized by significantly different iron abundances, spanning a total metallicity range of 1 dex (see also Origlia et al. 2011, 2013; Massari et al. 2014). The measured chemical patterns (Origlia et al. 2011, 2013) could be naturally explained in a scenario where Terzan 5 was originally much more massive than today $\left(\sim 10^{6} M_{\odot}\right.$; Lanzoni et al. 2010), thus to be able to retain the iron-enriched gas ejected by violent supernova explosions. The large number of type II supernovae required to explain the observed abundance patterns should have also produced a large population of neutron stars, mostly retained into the deep potential well of the massive proto-Terzan 5 and likely forming binary systems through tidal capture interactions. Finally, its large collision rate (Lanzoni et al. 2010), the largest among all Galactic globular clusters (see also Verbunt \& Hut 1987), could have highly promoted pulsar re-cycling processes, which can explain the production of the large population of MSPs/LMXBs now observed in the system.

Since the X-ray outburst detected by SWIFT is expected to also produce a significant enhancement of the optical luminosity (see Shahbaz \& Kuulkers 1998; Charles \& Coe 2006; Testa et al. 2012; Pallanca et al. 2014), we successfully applied for HST Director Discretionary Time to urgently survey the central region of Terzan 5 and thus provide new insights into this still unexplored phase of the LMXB-to-MSP path. Here we report on the identification of the optical counterpart to EXO 1745-248 obtained from the analysis of these images. 


\section{OBSERVATIONS AND DATA REDUCTION}

To search for the expected optical emission from EXO 1745-248 during its X-ray bursting phase, we submitted a HST Director Discretionary Time proposal (GO 14061, PI: Ferraro) asking for two orbits with the Advanced Camera for survey (ACS/WFC). The observations have been promptly performed on April 20, 2015, about one month into the X-ray outburst (continuing at time of writing). The dataset (hereafter Epoch 3, EP3) consists of $5 \times 398 \mathrm{~s}$ images in F606W, $5 \times 371 \mathrm{~s}$ images in F814W, and one short exposure per filter (50 s and $10 \mathrm{~s}$, respectively; these latter have not been used in the present work).

Previous optical images of Terzan 5 acquired with the same instrument in the same filters were already present in the HST Archive: GO 12933 (PI: Ferraro) performed on August 18th, 2013 (hereafter EP2), and GO 9799 (PI: Rich) performed on September 9th, 2003 (hereafter EP1). We already used these data to construct the deepest optical color-magnitude diagram (CMD) of Terzan 5 (see Ferraro et al. 2009; Lanzoni et al. 2010; Massari et al. 2012).

For the present study, all the datasets have been homogeneously analyzed, applying standard IRAF procedures for Pixel-Area-Map (PAM) correction on the (flc) images corrected for Charge Transfer Efficiency. The photometric analysis has been carried out by using the DAOPHOT package. For each image we modeled a Moffat point spread function (PSF) by using 150-200 bright and nearly isolated stars. Afterwards, we performed source detection in each image imposing a $3-\sigma$ threshold over the background level. By using all the sources detected and PSF fitted in at least 1 out of 2 images in EP1, and 3 out of 10 images in EP2 and EP3, we then created a catalog for every epoch. In spite of including sources detected in only one filter, such an approach allowed us to avoid losing very faint (but possibly real) objects, while safely discarding spurious detections, as cosmic rays and detector artifacts. The obtained master lists (one for every epoch) have been then used to identify the stellar sources in each single frame and the PSF model has been applied to derive the final magnitudes. As a final step, to build the EP2 and EP3 catalogs we considered all the stars with magnitude measured in both filters in at least 3 out of 5 images, while the EP1 catalog obviously consists of the objects detected in both the available images. For each star, the magnitudes estimated in different images of the same filter have been homogenized (see Ferraro et al. 1992) and their weighted mean and standard deviation have been finally adopted as the star magnitude and photometric error. The magnitude calibration to the VEGAMAG system has been performed by using the catalog by Massari et al. (2012) as reference.

To precisely determine the star coordinates, we first applied the equations reported by Meurer et al. (2002) and corrected the instrumental positions for the known geometric distortions affecting the ACS images. Through cross-correlation with the catalog of Massari et al. 
(2012), which had been placed onto the 2MASS system, we then obtained the absolute coordinates for each star, with a final astrometric accuracy of $\sim 0.2^{\prime \prime}$ in both right ascension and declination.
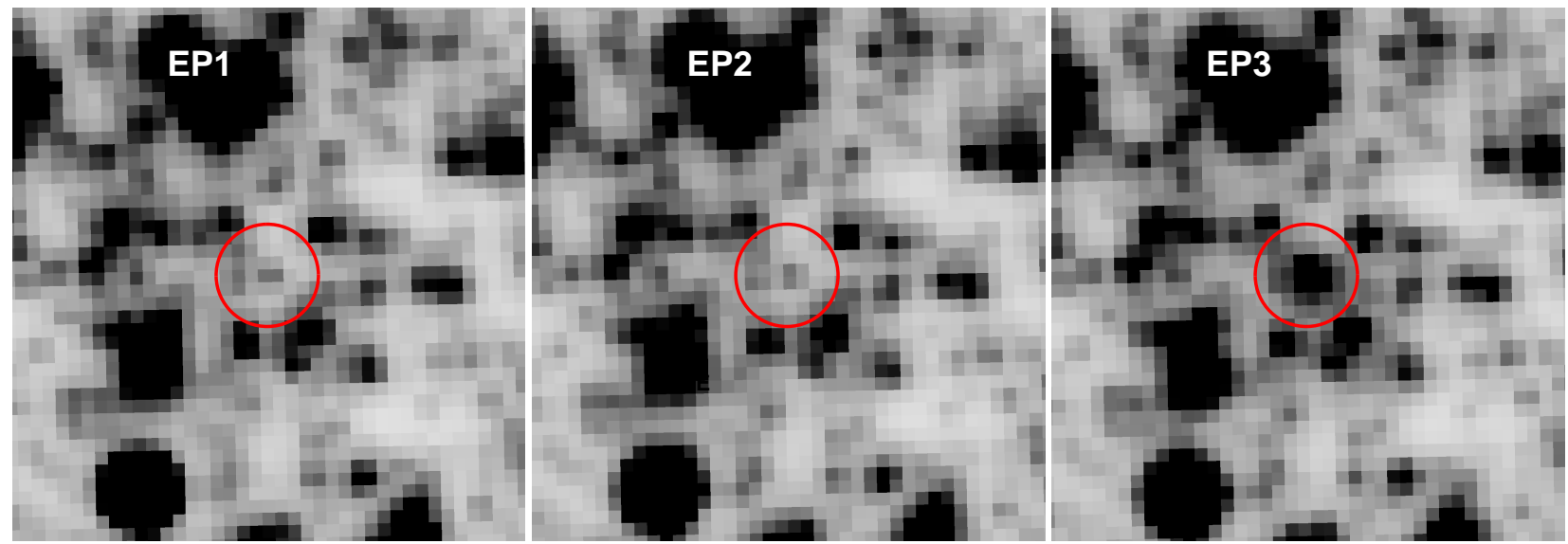

Fig. 1.- HST/ACS drz combined images of the $2^{\prime \prime} \times 2^{\prime \prime}$ region around EXO 1745-248, in the F814W filter, for the three epochs (EP1, EP2, EP3, from left to right, respectively). The source (highlighted with a red circle) is visible as a faint star during the quiescence epochs EP1 and EP2, while it is observed in an outburst stage during EP3. North is up, east is to the left.

\section{RESULTS}

The photometric analysis of our dataset in a region around the position of EXO 1745248 immediately revealed, in EP3, the presence of a bright star that was not visible in EP1 and EP2 images (see Figure 1). The comparison of the three epochs unequivocally identifies the bright object (hereafter COM-EXO 1745-248) as the optical counterpart to EXO 1745248. The absolute position of the optical source is $\mathrm{RA}(\mathrm{J} 2000)=17^{h} 48^{m} 05.23^{s}$, Dec $(\mathrm{J} 2000)=$ $-24^{\circ} 46^{\prime} 47.6^{\prime \prime}$. This is consistent at $1-\sigma$ with the VLA position quoted by Tremou et al. (2015, see the red and the green circles in Figure 2). Instead, the star previously suggested as the possible optical counterpart to this X-ray transient (Heinke et al. 2003) is located $\sim 0.7^{\prime \prime}$ to the west (cyan square in the figure).

After the astrometric transformations, the centroid position of the bright object in EP3 is within 0.05 pixels from the centroid of a fainter star clearly detected in EP1 and EP2 (see Figure 3). This could be the optical counterpart caught in quiescence. The probability that the true counterpart is a fainter, non detected star aligned (within 0.05 pixels) along the line 


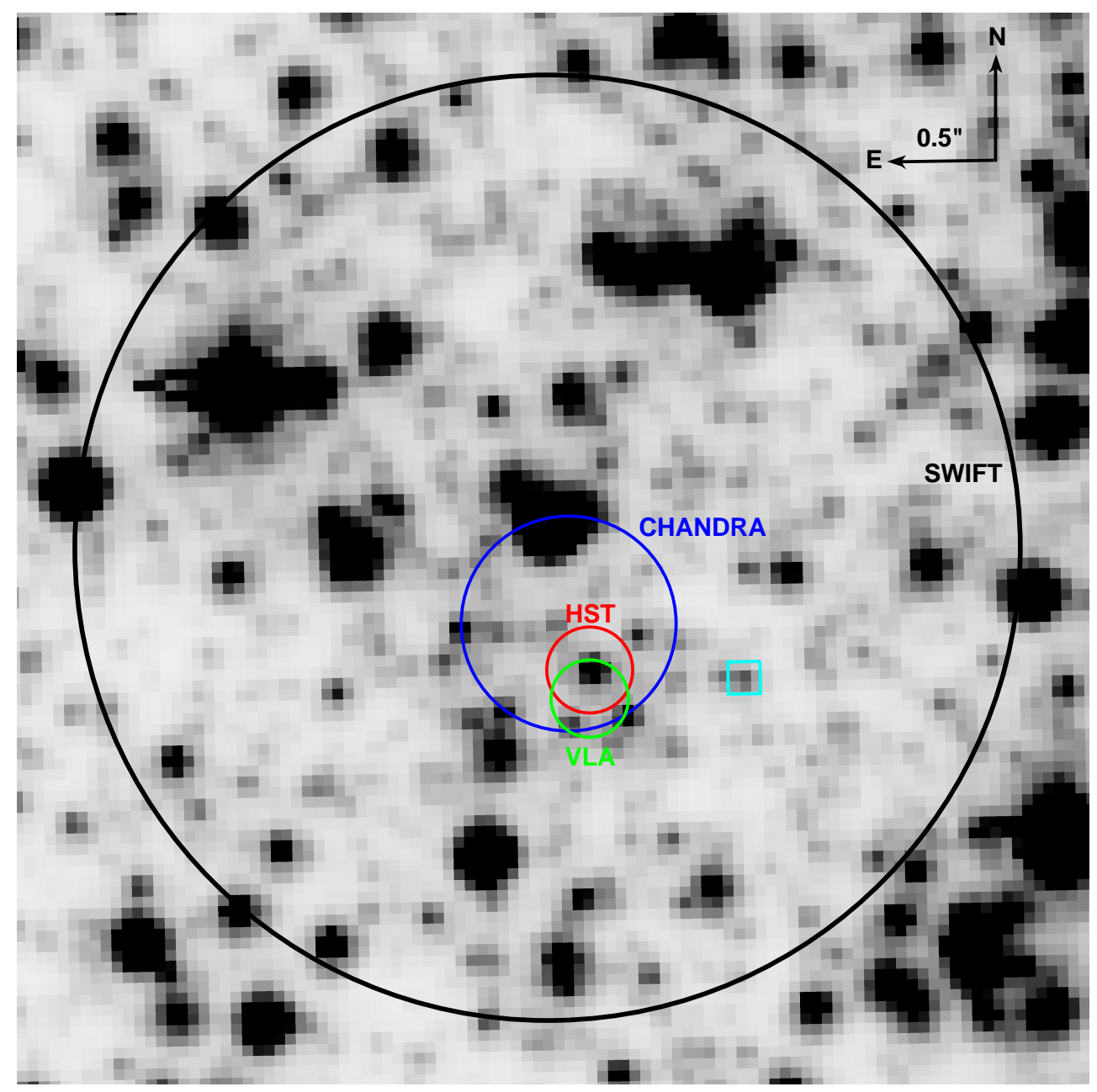

Fig. 2.- F814W-band drz combined image of the $5^{\prime \prime} \times 5^{\prime \prime}$ region around EXO 1745-248 in the EP3 exposure. The source positions and uncertainties obtained from the various observational campaigns are marked: the Swift/XRT 2.2" radius error circle is shown in black, the Chandra error circle in blue, the VLA measure in green, and the HST optical determination in red. The cyan square marks the star previously proposed (Heinke et al. 2003) as the possible optical counterpart to EXO 1745-248.

of sight is very low $(P \sim 0.4 \%) \sqrt[1]{1}$ In addition, the brightness profile of this star does not show

\footnotetext{
${ }^{1}$ To estimate the probability of a chance superposition with a star fainter than the proposed counterpart, the number of stars down to 5 magnitudes below the Turn Off level at the same distance $\left(\sim 5^{\prime \prime}\right)$ from the cluster center is needed. Since no data-set available for Terzan 5 reaches such a faint magnitude limit, we adopted as reference the luminosity function of 47 Tucanae derived from deep HST observations (Sarajedini et al. 2007). Star counts have been normalized to the number of Terzan 5 stars counted between
} 


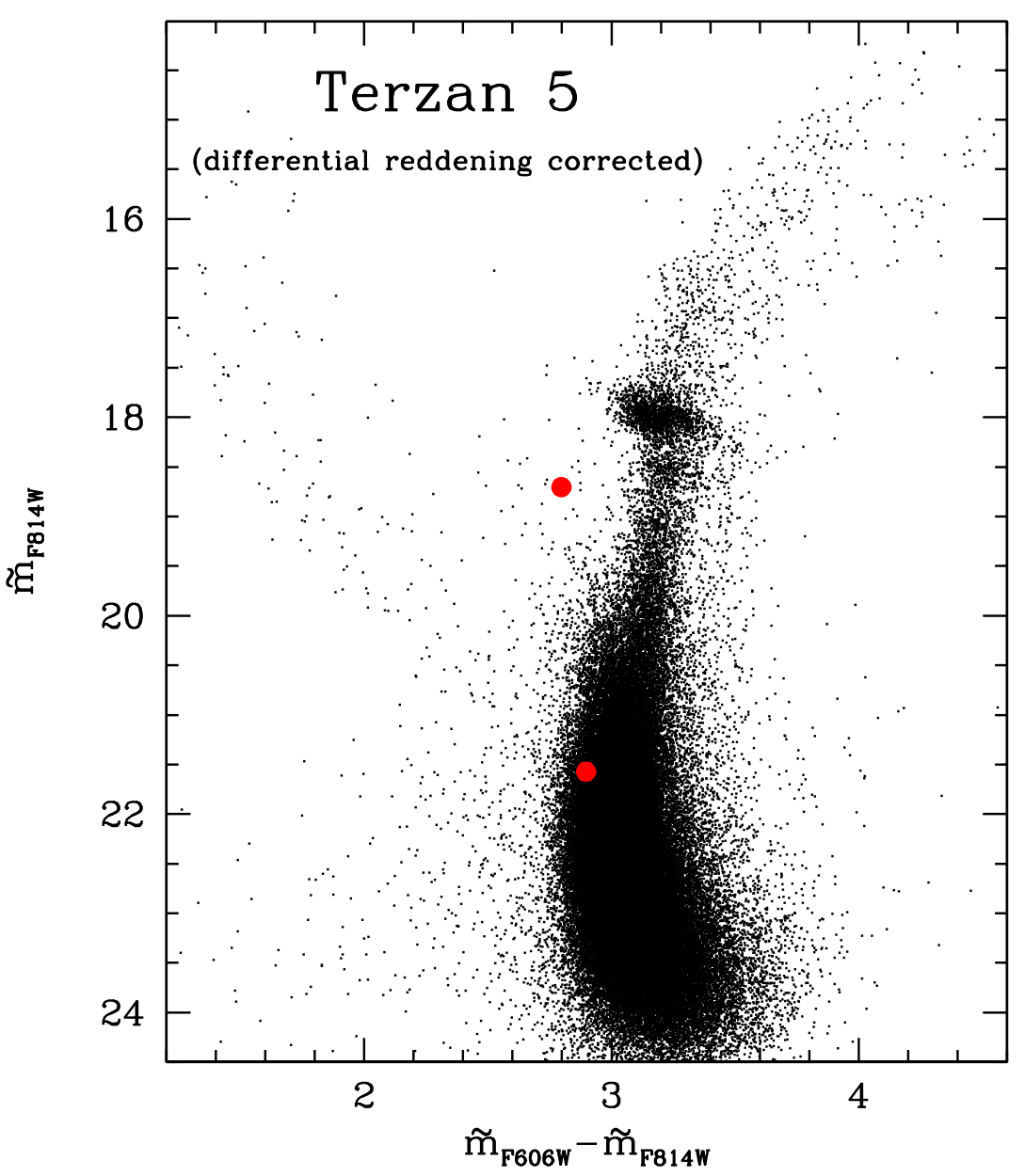

Fig. 3.- $\left(\mathrm{m}_{\mathrm{F} 814 \mathrm{~W}}, \mathrm{~m}_{\mathrm{F} 606 \mathrm{~W}}-\mathrm{m}_{\mathrm{F} 814 \mathrm{~W}}\right) \mathrm{CMD}$ of Terzan 5 corrected for differential reddening (according to Massari et al. 2012). The position of the optical counterpart to EXO 1745-248, in the outburst and in quiescence states, is marked with large red circles.

any significant deviation from symmetry, thus supporting the hypothesis that it is a single object. Hence, the most natural conclusion is that identified star is indeed the counterpart in quiescence. The identified object passed from observed magnitudes $\mathrm{m}_{\mathrm{F} 606 \mathrm{~W}}=24.74$ and $\mathrm{m}_{\mathrm{F} 814 \mathrm{~W}}=21.74$ during quiescence $(\mathrm{EP} 1$ and $\mathrm{EP} 2) 2$, to $\mathrm{m}_{\mathrm{F} 606 \mathrm{~W}}=21.77$ and $\mathrm{m}_{\mathrm{F} 814 \mathrm{~W}}=18.88$

the Turn Off level and two magnitudes above, in a ring of $2^{\prime \prime}$ width, centered at $5^{\prime \prime}$ from the center.

${ }^{2}$ The magnitudes of the star in the EP1 $\left(m_{\mathrm{F} 606 \mathrm{~W}}=24.7 \pm 0.1 ; m_{\mathrm{F} 814 \mathrm{~W}}=21.6 \pm 0.1\right)$ and $\mathrm{EP} 2\left(m_{\mathrm{F} 606 \mathrm{~W}}=\right.$ 


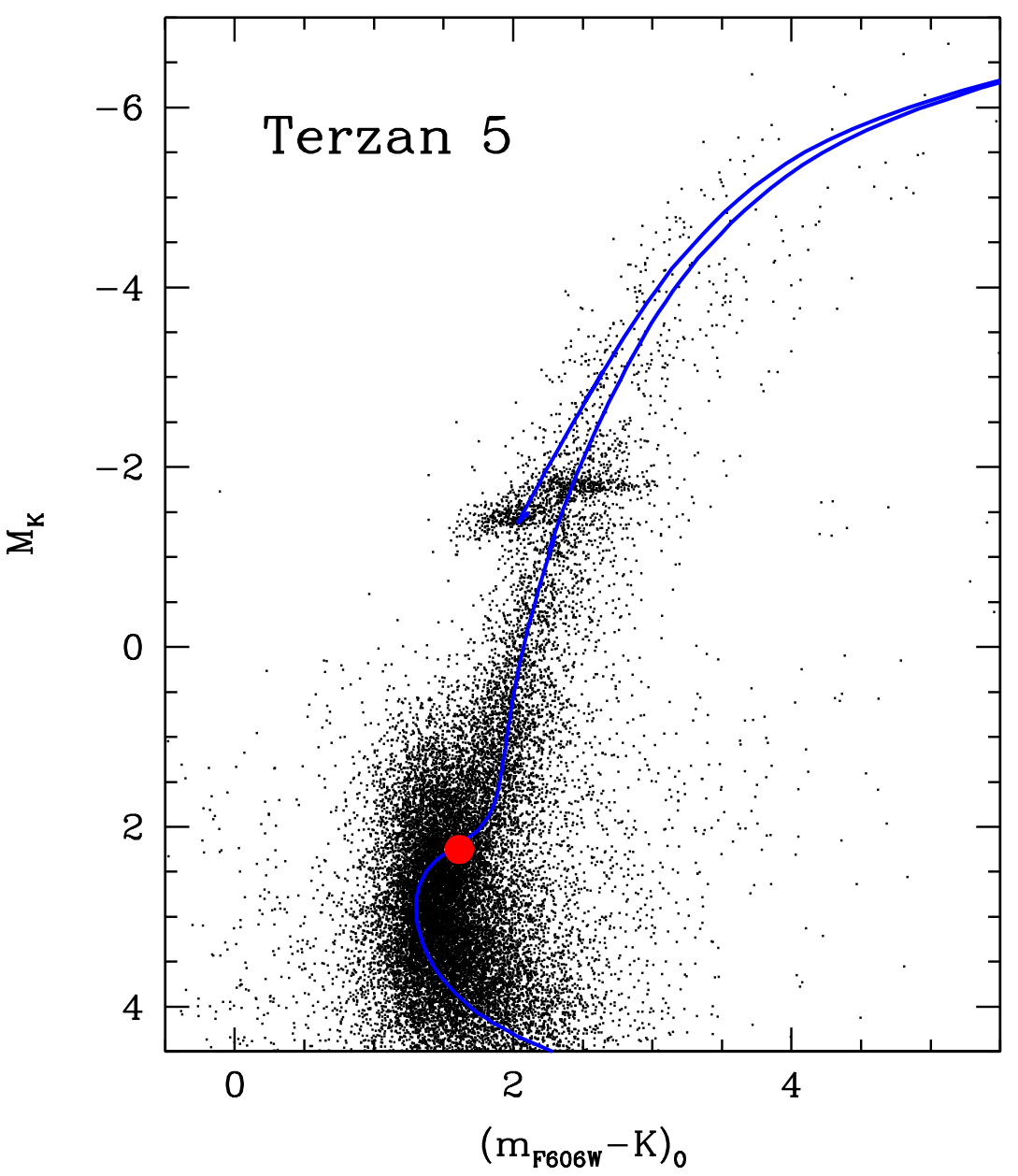

Fig. 4.- Absolute $\left(M_{K}, \mathrm{~m}_{\mathrm{F} 606 \mathrm{~W}}-K\right)_{0} \mathrm{CMD}$ of Terzan 5 obtained from a combination of HST/ACS and ESO/MAD observations. The position of COM-EXO 1745-248 in the quiescent state is marked with the large red circle. The blue line corresponds to a $12 \mathrm{Gyr}$ isochrone with $[\mathrm{Fe} / \mathrm{H}]=-0.3$ (from Girardi et al. 2010), well reproducing the main metal poor sub-population of Terzan 5.

in the outburst state (EP3), thus experiencing a brightening of 3 magnitudes (corresponding to a factor 16 in luminosity). Because of its location in the inner Galactic bulge, Terzan 5 is affected by a large extinction, with an average color excess $E(B-V)=2.38$ (Barbuy et al.

$\left.24.74 \pm 0.04 ; m_{\mathrm{F} 814 \mathrm{~W}}=21.7 \pm 0.1\right)$ quiescent stages are fully consistent within the errors. 
1998; Valenti et al. 2007), showing strong variations, up to $\delta E(B-V)=0.67$ mag, within the ACS field of view (Massari et al.|2012). We therefore applied the high-resolution differential reddening map obtained by Massari et al. (2012) to correct the observed magnitudes (in the following, the notation $\widetilde{\mathrm{m}}$ indicates magnitudes corrected for differential reddening). Figure 3 shows the position of COM-EXO 1745-248 in the differential reddening corrected CMD during the two states. We found $\widetilde{\mathrm{m}}_{\mathrm{F} 606 \mathrm{~W}}=24.47$ and $\widetilde{\mathrm{m}}_{\mathrm{F} 814 \mathrm{~W}}=21.57$ during EP1 and EP2, while $\widetilde{\mathrm{m}}_{\mathrm{F} 606 \mathrm{~W}}=21.50$ and $\widetilde{\mathrm{m}}_{\mathrm{F} 814 \mathrm{~W}}=18.70$ in EP3, corresponding to a small $(0.1 \mathrm{mag})$ color variation, which is however within the errors. No variability has been detected over the period of $\sim 50$ min covered by each HST orbit in EP3 and EP2. It is worth mentioning that EP3 data were acquired on April 20, 2015, almost simultaneously to the X-ray observations (Yan et al. 2015) suggesting that the system is transiting from a hard to a soft state.

An estimate of the orbital period of the system can be obtained by following (Shahbaz \& Kuulkers 1998), who reporte a relation between the orbital period and the $V$-band luminosity variation. Since we observe $\Delta V \sim 3$ mag in the case of EXO 1745-248, the orbital period turns out to be $P \sim 1.3$ days. On the other hand, for LMXBs van Paradijs \& McClintock (1994) proposed an empirical relation between the absolute $V$ magnitude in outburst and the parameter $\Sigma$, which depends on the ratio between the X-ray and the Eddington luminosities $\left(L_{\mathrm{X}} / L_{\mathrm{Edd}}\right)$ and the orbital period. By assuming $L_{\mathrm{X}} / L_{\mathrm{Edd}} \sim 0.5$ (Yan et al. 2015) and $M_{V}=1.37$ (in Johnson $V$ magnitude) for EXO 1745-248, we obtain $P \sim 0.1$ days. From these estimates, the orbital period of the system is likely to be between 1.3 and 0.1 days.

In order to more deeply investigate the nature of COM-EXO 1745-248 in the quiescence state under the assumption that the disk contribution to the observed magnitude is negligible, we identified the star in the K-band adaptive optics images obtained with ESO/MAD, used by Ferraro et al. (2009) to discover the two main multi-iron populations hidden in this system. We have first corrected the combined $\left(K, \mathrm{~m}_{\mathrm{F} 606 \mathrm{~W}}-K\right)$ CMD for differential reddening. Then we transformed it into the absolute plane by assuming the average color excess quoted above, and the distance modulus $(m-M)_{0}=13.87$ corresponding to a distance of 5.9 kpc (Valenti et al. 2007). The result is shown in Figure 4, where the position of COM-EXO 1745-248 in the quiescent state is marked. A more detailed characterization of the nature of COM-EXO 1745-248 is strongly hampered by the complexity of the stellar populations harbored in Terzan5. The comparison with a 12 Gyr old isochrone (Girardi et al. 2010) well reproducing the main metal-poor sub-population of Terzan 5 , at $[\mathrm{Fe} / \mathrm{H}]=-0.3$ dex 3

\footnotetext{
${ }^{3}$ As discussed in Massari et al. (2014), this population consists of $\sim 62 \%$ of the total, while a super-solar component at $[\mathrm{Fe} / \mathrm{H}]=+0.3$ dex accounts for $\sim 29 \%$, and an even metal poorer component, at $[\mathrm{Fe} / \mathrm{H}]=-0.8$ dex, recently detected by Origlia et al. (2013), corresponds to $\sim 5 \%$ of the total. The CMD plotted in Fig. 4 nicely shows two distinct red clumps at $M_{K}=-1.5$ and $M_{K}=-1.81$, corresponding to the two major
} 
suggests that COM-EXO 1745-248 could be a sub-giant branch (SGB) star. On the other hand the metal-rich sub-population could be significantly (a few Gyr) younger than the main metal poor component (see Ferraro et al. 2009). Thus, if COM-EXO 1745-248 belongs to the metal-rich component, it would be located below the SGB, in a position where companions to redback MSPs have been found (see, e.g., the case of COM-MSP6397A in Ferraro et al. 2001). Since no spectroscopic information on the metallicity of this star is available, both possibilities are equivalently valid. While in the case of redbacks any prediction on the stellar parameters based on the observed photometric properties can be difficult (see the case of COM-MSP6397A), this is possible for a SGB star belonging to the metal poor population. In this case, the following stellar parameters are obtained: mass $M=0.9 M_{\odot}$, effective temperature $T_{\text {eff }}=5440 \mathrm{~K}$, surface gravity $\log g=3.9$, and luminosity $\log L / L_{\odot}=0.35$. The corresponding stellar radius therefore is $R \sim 1.7 R_{\odot}$. Hence, by assuming that the star has completely filled its Roche Lobe and adopting a canonical value for the neutron star mass $\left(\sim 1.4 M_{\odot}\right)$, we derive an orbital separation $a \approx 5.2 R_{\odot}$ and a period $P_{\text {orb }} \sim 0.9 \mathrm{~d}$ for the binary system, fully in agreement with the range estimated above. We estimate that the radial velocity variations of such a binary system should have an amplitude of $\sim 170 \sin i \mathrm{~km}$ $\mathrm{s}^{-1}$ ( $i$ being the system inclination angle), which could be detectable through a dedicated spectroscopic follow-up.

\section{DISCUSSION AND CONCLUSIONS}

By using high-resolution images obtained with the HST/ACS during three different epochs, we have identified the optical counterpart to the neutron star transient EXO 1745248 in Terzan 5. With respect to the two previous epochs, this object shows a current brightening of $\sim 3$ magnitudes. In the quiescence state it is a sub giant branch star, i.e., an object that is experiencing its first envelope expansion, while evolving toward the red giant branch stage.

Very interestingly, the X-ray emission of EXO 1745-248 during quiescence was found to be highly variable both on short and long time-scales (Degenaar \& Wijnands 2012), and Linares et al. (2014) recently underlined that these properties are impressively similar to those observed for MSP-M28I, the system occasionally swinging between accretion-powered and rotation-powered emission (Papitto et al. 2013). This evidence suggests that EXO 1745248 could be another system belonging the rare class of objects caught shortly before the formation of a radio MSP, possibly in a stage immediately preceding the swinging phase in

sub-populations first discovered in the system by Ferraro et al. (2009). 
which MSP-M28I has been observed.

The characterization of EXO 1745-248 offers the opportunity of adding an additional link to the evolutionary chain connecting LMXBs to MSPs. In fact, from the analysis of the data currently available we can speculate that EXO1745-248 is experiencing the very early phase of the mass accretion stage, when an expanding star (a sub giant branch object) is filling its Roche Lobe and transferring material that eventually spins-up the neutron star. Indeed, the few outbursts in the X-ray occurring during this stage unambiguously indicate that heavy mass accretion on the neutron star is taking place. As time passes, the mass accretion rate will decrease and the system will enter into the phase characterized by a cyclic alternation between accretion- and rotation-powered emission, as observed in the case of MSP-M28I (Papitto et al. 2013). Thus, in the next future EXO1745-248 is expected to possibly start swinging between the LMXB and the MSP states. At later stages, when the neutron star has been sufficiently re-accelerated, the emission is powered by the rotating magnetic field, preventing further significant mass accretion. The system will be observable as a redback, i.e., a radio MSP with a highly perturbed companion, with the Roche Lobe filled and some material still falling toward the neutron star. Indeed, the prototype of this class of objects is MSP-A in NGC 6397 (Ferraro et al. 2001; see also Burderi et al. 2002), which shows a light curve dominated by tidal distortions (in agreement with the fact that the companion is a bloated, highly deformed star), prominent $\mathrm{H} \alpha$ lines (confirming the existence of diffuse gas outside the companion Roche Lobe; Sabbi et al. 2003), and peculiar chemical patterns (as expected for a deeply peeled star; Mucciarelli et al. 2013). The final fate will be that of a "canonical MSP", i.e., a NS in a binary system with a WD companion (the stripped core of the donor star) 4 .

We warmly thank the referee, Craig Heinke, for the careful reading of the manuscript and useful suggestions that help improving the paper. This research is part of the project Cosmic-Lab (web site: http://www.cosmic-lab.eu) funded by the European Research Council (under contract ERC-2010-AdG-267675).

\section{REFERENCES}

Altamirano, D., Krimm, H. A., Patruno, A., et al. 2015, The Astronomer's Telegram, 7240, 1

\footnotetext{
${ }^{4}$ Note that unexpected features have been observed also in this case, the most notable example being the optical variability of the WD companion to the pulsar 6752A in NGC 6752 (Cocozza et al. 2006).
} 
Bahramian, A., Heinke, C. O., Sivakoff, G. R., et al. 2014, ApJ, 780, 127

Bahramian, A., Altamirano, D., Heinke, C., et al. 2015, The Astronomer's Telegram, 7242, 1

Barbuy, B., Bica, E., \& Ortolani, S. 1998, A\&A, 333, 117

Bassa, C. G., Verbunt, F., van Kerkwijk, M. H., \& Homer, L. 2003, A\&A, 409, L31

Bhattacharya, D., \& van den Heuvel, E. P. J. 1991, Phys. Rep., 203, 1

Burderi, L., D’Antona, F., \& Burgay, M. 2002, ApJ, 574, 325

Charles, P. A., \& Coe, M. J. 2006, Compact stellar X-ray sources, 215

Cocozza, G., Ferraro, F. R., Possenti, A., \& D’Amico, N. 2006, ApJ, 641, L129

Cocozza, G., Ferraro, F. R., Possenti, A., et al. 2008, ApJ, 679, L105

Degenaar, N., \& Wijnands, R. 2012, MNRAS, 422, 581

Eckert, D., Del Santo, M., Bazzano, A., et al. 2013, The Astronomer's Telegram, 4925, 1

Edmonds, P. D., Gilliland, R. L., Heinke, C. O., Grindlay, J. E., \& Camilo, F. 2001, ApJ, $557, \mathrm{~L} 57$

Edmonds, P. D., Gilliland, R. L., Camilo, F., Heinke, C. O., \& Grindlay, J. E. 2002, ApJ, 579, 741

Ferraro, F. R., Fusi Pecci, F., \& Buonanno, R. 1992, MNRAS, 256, 376

Ferraro, F. R., Possenti, A., D’Amico, N., \& Sabbi, E. 2001, ApJ, 561, L93

Ferraro, F. R., Possenti, A., Sabbi, E., \& D’Amico, N. 2003, ApJ, 596, L211

Ferraro, F. R., Dalessandro, E., Mucciarelli, A., et al. 2009, Nature, 462, 483

Girardi, L., Williams, B. F., Gilbert, K. M., et al. 2010, ApJ, 724, 1030

Heinke, C. O., Edmonds, P. D., Grindlay, J. E., et al. 2003, ApJ, 590, 809

Heinke, C. O., Wijnands, R., Cohn, H. N., et al. 2006, ApJ, 651, 1098

Kuulkers, E., den Hartog, P. R., in't Zand, J. J. M., et al. 2003, A\&A, 399, 663

Lanzoni, B., Ferraro, F. R., Dalessandro, E., et al. 2010, ApJ, 717, 653 
Linares, M., Bahramian, A., Heinke, C., et al. 2014, MNRAS, 438, 251

Linares, M., Chakrabarty, D., Marshall, H., et al. 2015, The Astronomer's Telegram, 7247, 1

Massari, D., Mucciarelli, A., Dalessandro, E., et al. 2012, ApJ, 755, L32

Massari, D., Mucciarelli, A., Ferraro, F. R., et al. 2014, ApJ, 795, 22

Meurer, G.R. et al. 2002, in Proc. 2002 HST Calibration Workshop, ed. S. Arribas et al. (Baltimore:STScI), 65

Mucciarelli, A., Salaris, M., Lanzoni, B., et al. 2013, ApJ, 772, L27

Origlia, L., Rich, R. M., Ferraro, F. R., et al. 2011, ApJ, 726, L20

Origlia, L., Massari, D., Rich, R. M., et al. 2013, ApJ, 779, LL5

Pallanca, C., Dalessandro, E., Ferraro, F. R., et al. 2010, ApJ, 725, 1165

Pallanca, C., Dalessandro, E., Ferraro, F. R., Lanzoni, B., \& Beccari, G. 2013, ApJ, 773, 122

Pallanca, C., Ransom, S. M., Ferraro, F. R., et al. 2014, ApJ, 795, 29

Papitto, A., D'Aì, A., Motta, S., et al. 2011, A\&A, 526, L3

Papitto, A., Ferrigno, C., Bozzo, E., et al. 2013, Nature, 501, 517

Ransom, S. M., Hessels, J. W. T., Stairs, I. H., et al. 2005, Science, 307, 892

Sabbi, E., Gratton, R., Ferraro, F. R., et al. 2003, ApJ, 589, L41

Sarajedini, A., Bedin, L. R., Chaboyer, B., et al. 2007, AJ, 133, 1658

Shahbaz, T., \& Kuulkers, E. 1998, MNRAS, 295, L1

Sigurdsson, S., Richer, H. B., Hansen, B. M., Stairs, I. H., \& Thorsett, S. E. 2003, Science, 301, 193

Strohmayer, T. E., Markwardt, C. B., Pereira, D., \& Smith, E. A. 2010, The Astronomer's Telegram, 2946, 1

Testa, V., di Salvo, T., D'Antona, F., et al. 2012, A\&A, 547, A28

Tremou, E., Sivakoff, G., Bahramian, A., et al. 2015, The Astronomer's Telegram, 7262, 1 
Valenti, E., Ferraro, F. R., \& Origlia, L. 2007, AJ, 133, 1287

van Paradijs, J., \& McClintock, J. E. 1994, A\&A, 290, 133

Verbunt, F., \& Hut, P. 1987, in Proc. IAU Symp. 125, The Origin and Evolution of Neutron Stars, ed. D. J. Helfand \& J.-H. Huang (Dordrecht: Reidel), 187

White, N. E., Kaluzienski, J. L., \& Swank, J. H. 1984, American Institute of Physics Conference Series, 115, 31

Wijnands, R., \& van der Klis, M. 1998, Nature, 394, 344

Yan, Z., Lin, J., Yu, W., Zhang, W., \& Zhang, H. 2015, The Astronomer's Telegram, 7430, 1 